\title{
GLOBAL BEHAVIOR OF CURVES IN A SPACE OF POSITIVE CURVATURE
}

\author{
B. V. DEKSTER ${ }^{1}$
}

\begin{abstract}
It is well known that any geodesic in a complete noncompact space of positive curvature goes to infinity. In this paper, we prove that this is true for more general curves and estimate how fast they go to infinity in terms of their curvature and curvature of the space.
\end{abstract}

0. Introduction.

0.1. All manifolds, submanifolds and curves here are assumed to be of class $C^{\infty}$. A curve parametrized by its arc length is said to be normal.

Let $\gamma:[0, \infty) \rightarrow R^{n}$ be a normal curve, $\xi(s)$ be its curvature, and

$$
\Xi(s) \stackrel{\text { def }}{=} \int_{0}^{s} \xi(x) d x, \quad s \in[0, \infty)
$$

It is well known that $\gamma$ goes to $\infty$, i.e. escapes from any compact part of the space, if (1) $\xi(s) \underset{s \rightarrow \infty}{\rightarrow} 0$ or if (2) $\Xi(\infty)<\infty$. In a Riemannian space, a curve satisfying (1) or (2), even a geodesic, may stay in a compact part of the space. However, Gromoll and Mayer have proved that any geodesic in a complete noncompact $n$-dimensional, $n \geq 2$, Riemannian manifold $N$ of positive sectional curvature goes to $\infty$ (see [5, p. 85, Lemma 6]). The following simple remark extends the above criteria (1) and (2) of going to $\infty$ to curves in $N$.

0.2 . REMARK. Let $\gamma:[0, \infty) \rightarrow N$ be a normal curve, $\xi(s)$ be its curvature and $\Xi(s) \stackrel{\text { def }}{=} \int_{0}^{s} \xi(t) d t, s \in[0, \infty)$. Then

(i) $\gamma$ goes to $\infty$ if $\lim _{s \rightarrow \infty} \xi(s)=0$;

(ii) $\gamma$ goes to $\infty$ if $\varliminf_{s \rightarrow \infty} \Xi(s) / s=0$.

(In particular, $\gamma$ goes to $\infty$ if $\Xi(\infty)<\infty$.)

The statement (i) is mentioned just because of its clearness. In fact, (i) is embraced by (ii) since the condition $\xi(s) \underset{s \rightarrow \infty}{\rightarrow} 0$ implies that $\varliminf_{s \rightarrow \infty} \Xi(s) / s=0$. Remark 0.2 can be easily proved on the basis of the above-mentioned Lemma 6 in [5] and the fact that a set of (general) curves with limited lengths and total curvatures lying in a compact part of $N$ is compact. (This possibility was pointed out to the author by M. L. Gromov.) An even shorter proof will be produced in 3.4. The proof in 3.4 involves a somewhat complicated construction. But this construction is used elsewhere in the paper.

0.3 . Another natural question is at what rate curves go to $\infty$. Let $\gamma$ be as in 0.1 and let $s(t)$ be the maximum number such that $\left.\gamma\right|_{[0, s(t)]}$ finds room in the closed ball of radius $t$ centered at $\gamma(0)$. Then it seems reasonable to measure this rate by the

Received by the editors November 3, 1981. Presented to the 87th Annual Meeting of the AMS in San Francisco on January 10, 1981.

1980 Mathematics Subject Classification. Primary 53C40.

${ }^{1}$ Supported partly by an NSF Grant. 
quantity $\lim _{t \rightarrow \infty} s(t) / t$. For a ray, say, this limit is 1 , while for a cylindrical spiral it is greater than 1. We are not aware of published estimates of that limit (for $R^{n}$ ). Such estimates follow easily from suitable upper bounds of the length of a curve within a ball of radius $t$. Suppose, for example, that $B(\infty)<\cos ^{-1}(-1 /(n-1))$, and that all the vectors $\dot{\gamma}(s), 0 \leq s<\infty$, point into the same halfspace. Then Resetnjak's Theorem [6, p. 262] implies that

$$
s(t) \leq t / \sqrt{\frac{1+(n-1) \cos \Xi(s(t))}{n}},
$$

and, consequently,

$$
\varlimsup_{t \rightarrow \infty} \frac{s(t)}{t} \leq \sqrt{\frac{n}{1+(n-1) \cos \Xi(\infty)}} .
$$

To the best of our knowledge, for the space $N$, analogous upper bounds were found only recently (see [2 and 3]). Moreover, these upper bounds deal with a domain different from a metric ball. Nevertheless [2 and 3], along with [4], enable us to estimate the rate of going to $\infty$ in $N$ (Theorems 1.4, 1.5).

The following corollary illustrates these theorems.

0.4. Corollary. Let $p \in N$ and let $B_{t}(p)$ be the closed metric ball of radius $t$ centered at $p$. Denote by $k(t)$ the minimum sectional curvature in $B_{t}(p) .(k(t) \rightarrow 0$ as $t \rightarrow \infty$ since $M$ is noncompact.) Suppose that

$$
h_{\beta} \stackrel{\text { def }}{=} \varlimsup_{t \rightarrow \infty} \pi /\left(t^{\beta} \sqrt{k(t)}\right)
$$

is finite for some $\beta(>0)$. Let $g: R \rightarrow N$ be a normal geodesic. Denote by $\rho(\cdot, \cdot)$ the distance in $N$. For $t>\rho(p, g(0))$, denote by $s(t)$ the length of the maximum segment $I \subset R$ such that $I \ni 0$ and $g(I) \subset B_{t}(p)$. Then $\beta \geq 1, h_{\beta}$ does not depend on the point $p$, and

$$
\varlimsup_{t \rightarrow \infty} s(t) / t^{\beta} \leq h_{\beta} .
$$

Further, let $\gamma:[0, \infty) \rightarrow N$ be a normal curve with curvature $\xi(s)$. Suppose there exist numbers $A>0$ and $\alpha \in(0,1)$ such that $\int_{0}^{s} \xi(x) d x \leq A s^{\alpha}$ for sufficiently large s. (By Remark 0.2(ii), $\gamma$ goes to $\infty$.) For $t>\rho(p, \gamma(0))$, denote by $\lambda(t)$ the maximum number such that $\gamma([0, \lambda(t)]) \subset B_{t}(p)$. Then

$$
\varlimsup_{t \rightarrow \infty} \lambda(t) / t^{(2 \beta-1) /(1-\alpha)} \leq\left[4 \sqrt{2} A h_{\beta}^{2}(2 \beta-1) / \pi^{3}\right]^{1 /(1-\alpha)} .
$$

Moreover, the upper limits in (0.4.1) and (0.4.2) do not depend on the choice of the point $p \in N$.

This corollary is proved in 3.7.

\section{Summary and discussion of the results.}

1.1. In 0.4., we used the class of power functions as a scale in estimating the rate of going to $\infty$. We shall now use for that purpose a somewhat larger class $Z$, consisting of functions $z(t), t \in(0, \infty)$, satisfying

$$
z(t)>0, \quad z(t) \underset{t \rightarrow \infty}{\rightarrow} \infty, \quad \frac{z(R(t))}{z(t)} \underset{t \rightarrow \infty}{\rightarrow} 1
$$


for any positive function $R(t)$ such that

$$
\frac{R(t)}{t} \underset{t \rightarrow \infty}{\rightarrow} 1 \text {. }
$$

(Then, say, $\alpha(t) \cdot t^{\beta} \in Z$ for any $\alpha(t)$ satisfying $0<\alpha_{1}<\alpha(t)<\alpha_{2}$ and any $\beta>0 ; \ln (t+1) \in Z$; but $e^{t} \notin Z$.)

1.2. Let $B_{t}(p)$ and $k(t)$ be as in Corollary 0.4. By Theorem 1.6 in [4], the function $k(t)$ has a convexity function $\epsilon(t), t \in(a, \infty) .(\epsilon(t)$ is the minimal positive solution of the equation $\phi^{\prime}=-\phi^{2}-k(t)$, see [4] for details.) Put

$$
\Phi_{1}(z)=\varlimsup_{t \rightarrow \infty} \frac{2}{z \sqrt{k}} \tan ^{-1} \frac{\sqrt{k}}{\epsilon} ; \quad \Phi_{2}(z)=\varlimsup_{t \rightarrow \infty} \frac{2}{z \epsilon} ; \quad \Phi_{3}(z)=\varlimsup_{t \rightarrow \infty} \frac{\pi}{z \sqrt{k}} .
$$

LEMMA. If $z \in Z$ and $\Phi_{j}(z), j \in\{1,2,3\}$, is finite then $\Phi_{j}(z)$ does not depend on the choice of the point $p \in N$.

The proof is given in 3.1.

(Thus, the numbers $\Phi_{j}(z)$ with $z \in Z$ characterize the space $N$. Our results are expressed in terms of the $\Phi_{j}$.)

1.3. Notice that a particular finite number $\Phi_{j}(y)$ with $y \notin Z$ can depend on $p$. An example of this is $\Phi_{3}\left(e^{t / 2}\right)$ for the surface $M_{0}$ (see [4, Remark 1.6]) with an exponentially decreasing $k(t)$. Obviously, $\Phi_{3}(z)$ for such an $M_{0}$ is always infinite if $z \in Z$.

Since $\tan ^{-1}(x)<x$ for $x>0$, and $\tan ^{-1}(x)<\pi / 2$, we have $\Phi_{1} \leq \Phi_{2}, \Phi_{1} \leq$ $\Phi_{3}$.

1.4. THEOREM. Let $p \in N, B_{t}(p)$ be the closed ball of radius $t$ centered at $p$ and let $g: R \rightarrow N$ be a normal geodesic. For $t>\rho(p, g(0))$ denote by $s(t)$ the length of the maximum segment $I \in R$ such that $I \ni 0$ and $g(I) \subset B_{t}(p)$. Suppose $\Phi_{j}(z)<\infty$ for some $z \in Z$ and $j \in\{1,2,3\}$. Then

$$
\varlimsup_{t \in \infty} \frac{s(t)}{z(t)} \leq \Phi_{j}(z)
$$

Moreover, the upper limit in (1.4.1) does not depend on $p \in N$.

Theorem 1.4 is proved in 3.6.

The estimate (1.4.1) is not exact.

Obviously, $s(t) \geq t-\rho(p, g(0))$. Therefore, by (1.4.1), $\Phi_{j}\left(t^{\beta}\right)=\infty$ for any $\beta \in(0,1), j \in\{1,2,3\}$.

1.5. THEOREM. Let $B_{t}(p)$ be as in Theorem 1.4. Let $\gamma:[0, \infty) \rightarrow N$ be a normal curve and $\xi(s)$ be its curvature. Suppose there exist $A>0$ and $\alpha \in[0,1)$ such that

$$
\Xi(s) \stackrel{\text { def }}{=} \int_{0}^{s} \xi(x) d x \leq A s^{\alpha}
$$

for sufficiently large s. (By Remark $0.2, \gamma$ goes to $\infty$.) For $t>\rho(p, \gamma(0))$, denote by $\lambda(t)$ the maximum number such that $\gamma([0, \lambda(t)]) \subset B_{t}(p)$. Suppose $\Phi_{2}(z)<\infty$ for some $z \in Z$. Then

$$
\varlimsup_{t \rightarrow \infty} \frac{\lambda(t)}{z(t)} \leq\left\{\begin{array}{l}
{\left[(2 \sqrt{2} A / \pi) \cdot \Phi_{2}\left(z^{1-\alpha}\right)\right]^{1 /(1-\alpha)} \quad \text { if } \alpha \in(0,1),} \\
(2 \sqrt{2} A / \pi+\sqrt{2}) \cdot \Phi_{2}(z) \quad \text { if } \alpha=0 .
\end{array}\right.
$$

Moreover, the upper limit in (1.5.2) does not depend on $p \in N$. 
The proof is given in 3.5.

Notice that the case $\alpha \geq 1$ is not of interest since, in that case, any periodic curve (obviously, such a curve does not go to $\infty$ ) satisfies $\Xi(s) \leq A s \leq A s^{\alpha}$ for large $s$.

The estimate (1.5.2) is not exact.

1.6. The idea of the proofs of the above theorems is as follows. For a point $p \in N$, we construct the expanding family of Gromoll and Meyer's totally convex sets $C_{t}(p)$, satisfying $N=\bigcup_{t \geq 0} C_{t}(p)$, as described in [1, Proposition 1.3]. The results of [4] allow us to estimate the degree of convexity of $\partial C_{t}(p)$ in terms of the minimum sectional curvature in $C_{t}(p)$ (see [4, Theorems 1.11 and 1.13]). Now, using the results of [2 and 3] one can estimate from above the length of the part of an infinite curve that finds room in $C_{t}(p)$. This upper bound is expressed in terms of the above-mentioned degree and therefore, finally, in terms of the minimal sectional curvature in $C_{t}(p)$. Passage to the limit yields the desired asymptotics.

We use in this reasoning the minimum sectional curvature in $C_{t}(p)$ instead of $B_{t}(p)$. However, this turns out to be of little consequence since these sets are close for large $t$ (see [4, Remark 1.2]).

Another difficulty is connected with the application of the estimates of $[2,3]$ to the set $C_{t}(p)$. For that, one needs to generalize the results of $[2,3]$ to the case of a region with a nonsmooth boundary. This generalization is given in $\S 2$.

The author is indebted to Ju. D. Burago for the general idea of using the filtration $C_{t}(p)$ and the estimates in $[2,3]$ for a study of the global behavior of curves. Partly, this idea was realized in [4].

\section{A normal region with a nonsmooth boundary.}

2.1. In $\S 2, N$ denotes an arbitrary Riemannian manifold of dimension $n \geq 2$. A set $S \subset N$ will be called $[\kappa, y]$-convex, $\kappa, y>0$, if any $C^{\infty}$-curve of the length $<y$ and of curvature $<\kappa$ having its ends in $S$ belongs to $S$.

A set $S \subset N$ will be called $[\kappa]$-convex, $\kappa>0$, if for any $x \in(0, \kappa)$, one can find $y>0$ such that the set $S$ is $[x, y]$-convex.

THEOREM. Let $D \subset N$ be a compact $[\kappa, y]$-convex region with a nonempty boundary $\partial D$. Then for any $\epsilon>0$ one can construct a compact region $D^{\epsilon}$ such that

1. $D \subset D^{\epsilon} \subset D_{\epsilon}$ where $D_{\epsilon}$ is the $\epsilon$-neighbourhood of $D$;

2. $\partial D^{\epsilon}$ is a nonempty $C^{\infty}$-surface;

3. The normal curvatures of $\partial D^{\epsilon}$ on the side of the inner normal are not less than $\kappa-C(\epsilon)$ where $C(\epsilon) \rightarrow 0$ as $\epsilon \rightarrow 0$.

To our knowledge, this theorem has been proved by V. A. Sharafutdinov in his dissertation (kept in Novosibirsk). Although the dissertation is not easily available, we omit the proof of the theorem since it does not involve especially complicated ideas. The proof we have devised (and written down) is too long to be reproduced here.

2.2. Let $M \subset N$ be a compact $[\kappa]$-convex region, $\kappa>0$, with a nonempty boundary $\partial M$. Suppose the sectional curvature $\geq k_{s}>-\kappa^{2}$ in $M$. Then $M$ is said to be a generalized normal region.

As in $[4,1.4]$, we assign to $M$ a circle $M_{0}$ on the $k_{s}$-plane whose circumference $\partial M_{0}$ has geodesic curvature $\kappa$. $M_{0}$ exists because $k_{s}>-\kappa^{2}$. Its radius $R_{0}=$ $R_{0}\left(\kappa, k_{s}\right)$ is given by formula (1.4) in [4]. 
THEOREM. The length of any curve $\gamma$ with maximum curvature $\chi \in[0, \kappa)$ that lies in a generalized normal region $M$ does not exceed the length of a circular arc with curvature $\chi$ that lies in $M_{0}$ and whose ends are opposite points of $\partial M_{0}$.

2.3. Proof. Let $x \in(0, \kappa)$ satisfy $k_{s}>-x^{2}, \chi<x$, and let $y=y(x)$ be such that $M$ is $[x, y]$-convex. By Theorem 2.1, for any $\epsilon>0$ there exists a compact region $M^{\epsilon}$ satisfying $M_{\epsilon} \supset M^{\epsilon} \supset M \supset \gamma$ with a nonempty $C^{\infty}$-boundary $\partial M^{\epsilon}$ whose normal curvatures are $\geq x-C(\epsilon)$. For sufficiently small $\epsilon, \chi<x-C(\epsilon)$ and a lower bound $k_{s}(\epsilon)$ of the sectional curvatures in $M^{\epsilon}$ can be chosen such that $k_{s}(\epsilon)>-(x-C(\epsilon))^{2}$. We also may suppose that $k_{s}(\epsilon) \rightarrow k_{s}$ as $\epsilon \rightarrow 0$. By the corollary in [2, p. 103], the length of $\gamma \subset D^{\epsilon}$ does not exceed the length of a circular arc of curvature $\chi$ connecting opposite points on the circumference of a circle of radius $R_{0}\left(x-C(\epsilon), k_{s}(\epsilon)\right)$ in the $k_{s}(\epsilon)$-plane. The passage to the limit in this inequality, as $\epsilon \rightarrow 0$ and then as $x \rightarrow \kappa$, completes the proof.

2.4. THEOREM. Theorem 1.6 in [3] remains true if $M$ in [3] is taken to be a generalized normal region.

The proof of this theorem is similar to the proof in 2.3, except that we apply Theorem 1.6 in [3] instead of the corollary in [2].

3. Rate at which curves go to $\infty$.

3.1. PROOF OF LemMA 1.2. Let $p^{*} \in N, \rho\left(p, p^{*}\right)=\Delta>0$ and $t>\Delta$. In what follows, the notation $X^{*}$ has the same meaning for the point $p^{*}$ that $X$ has for $p$. By the triangle inequality, $B_{t-\Delta}(p) \subset B_{t}\left(p^{*}\right) \subset B_{t+\Delta}(p)$. Therefore,

$$
k(t-\Delta) \geq k^{*}(t) \geq k(t+\Delta), \quad t>\Delta \text {. }
$$

Put

$$
\tilde{k}(t)=\left\{\begin{array}{l}
k(t), \quad t \geq 0 \\
k(0), \quad t<0
\end{array}\right.
$$

$k^{-}(t)=\tilde{k}(t-\Delta), k^{+}(t)=\tilde{k}(t+\Delta)$ and denote by $\epsilon^{-}:\left(a^{-}, \infty\right) \rightarrow R, \epsilon^{+}:\left(a^{+}, \infty\right)$ $\rightarrow R$ the convexity functions for $k^{-}, k^{+}$(see $\left.[4,1.4]\right)$. The set of solutions of the equation $\phi^{\prime}=-\phi^{2}-k^{-}(t)\left(\phi^{\prime}=-\phi^{2}-k^{+}(t)\right)$ is the result of "a shift" of the solutions of the equation $\phi^{\prime}=-\phi^{2}-\tilde{k}(t)$ through a distance $\Delta(-\Delta)$ parallel to the $t$-axis. Therefore, $a^{-}=a+\Delta$, $a^{+}=a-\Delta$ and $\epsilon^{-}(t)=\epsilon(t-\Delta)$, $\epsilon^{+}(t)=\epsilon(t+\Delta)$ for $t>a^{-}=a+\Delta$. This is also true for $t>\Delta$ since $a \leq 0$, see $[4,1.5,1.6]$. By (3.1.1) and $\left[4\right.$, Lemma 3.9] we have $\epsilon^{-}(t) \geq \epsilon^{*}(t) \geq \epsilon^{+}(t)$, i.e.

$$
\epsilon(t-\Delta) \geq \epsilon^{*}(t) \geq \epsilon(t+\Delta), \quad t>\Delta .
$$

Using (3.1.1), (3.1.2) and the fact that $(1 / x) \tan ^{-1}(x / y)$ is a decreasing function of $x$ in the region $x>0, y>0$, we find that

$$
\begin{gathered}
\frac{1}{z(t) \sqrt{k(t-\Delta)}} \tan ^{-1} \frac{\sqrt{k(t-\Delta)}}{\epsilon(t-\Delta)} \leq \frac{1}{z(t) \sqrt{k^{*}(t)}} \tan ^{-1} \frac{\sqrt{k^{*}(t)}}{\epsilon^{*}(t)} \\
\leq \frac{1}{z(t) \sqrt{k(t+\Delta)}} \tan ^{-1} \frac{\sqrt{k(t+\Delta)}}{\epsilon(t+\Delta)}
\end{gathered}
$$


Let us now replace $1 / z(t)$ in the left-hand part by $(z(t-\Delta) / z(t)) \cdot(1 / z(t-\Delta))$ and in the right-hand part by $(z(t+\Delta) / z(t)) \cdot(1 / z(t+\Delta))$. Obviously,

$$
\lim _{t \rightarrow \infty} \frac{z(t-\Delta)}{z(t)}=\lim _{t \rightarrow \infty} \frac{z(t+\Delta)}{z(t)}=1
$$

because $z \in Z$ and $(t-\Delta) / t \rightarrow 1,(t+\Delta) / t \rightarrow 1$. Passing to the upper limit in (3.1.3) as $t \rightarrow \infty$, we get $\Phi_{1}(z) \stackrel{t \rightarrow \infty}{\leq} \Phi_{1}^{*}(z) \leq \Phi_{1}(z)$, i.e. $\Phi_{1}^{*}(z)=\Phi_{1}(z)$.

The equalities $\Phi_{2}^{*}(z)=\Phi_{2}(z)$ and $\Phi_{3}^{*}(z)=\Phi_{3}(z)$ are proved similarly.

3.2. For the other proofs we need a few general remarks. Let $p \in N$ and $C_{t}(p)$, $t \geq 0$, be the expanding family of Gromoll and Meyer's compact totally convex sets constructed with the help of the rays emanating from $p$ as described in $[1$, Proposition 1.3]. Then $p \in \partial C_{0}, \bigcup_{t>0} C_{t}(p)=N$. By Remark 1.2 in [4], $C_{t}(p) \supset$ $B_{t}(p)$. By [1, pp. 417-418], it follows that the set $C_{t}(p), t>0$, is a compact region. (Note that $C_{0}(p)$ can be a point.) By Theorem 1.11 in [4], $C_{t}(p)$ is an $[\epsilon(R)]$-convex region where $R=R(t)=\max _{q \in C_{t}(p)} \rho(q, p)$. By $[4,1.4], \epsilon(R)>0$. Since $C_{t}(p) \subset$ $B_{R(t)}(p)$, the sectional curvatures there are $\geq k(R)$. Since $k(R)>0>-\epsilon^{2}(R)$, it follows that $C_{t}(p)$ is a generalized normal region (see 2.2).

We assign to $C_{t}(p)$ two circles: a circle $M_{0}$ of radius $R_{0}(\epsilon(R), k(R)$ ) (see [4, (1.4)]) in the $k(R)$-plane and a circle $M_{0}^{\prime}$ of radius $R_{0}(\epsilon(R), 0)(=1 / \epsilon(R))$ in the Euclidean plane.

3.3. By Proposition 4.2 in [3], the longest locally convex curve in $M_{0}^{\prime}$ with total curvature $\pi / 2$ is a polygonal line $A B C$ such that $A, B, C \in \partial M_{0}^{\prime}, A B=B C$ and the angle $A B C=\pi / 2$. Its length is $2 \sqrt{2} / \epsilon(R)$.

Let $\gamma:[0, \infty) \rightarrow N$ be a normal curve with curvature $\xi(s)$. By Theorem 2.4, if $\left.\gamma\right|_{[a, b]}$ lies in $C_{t}(p)$ and $\int_{a}^{b} \xi(x) d x \leq \pi / 2$, then $b-a \leq 2 \sqrt{2} / \epsilon(R)$. Therefore, if $\left.\gamma\right|_{[0, s]}$ lies in $C_{t}(p), s>0$, then

$$
s \leq\left(\frac{\Xi(s)}{\pi / 2}+1\right) \frac{2 \sqrt{2}}{\epsilon(R)} \text { where } \Xi(s)=\int_{0}^{s} \xi(x) d x .
$$

3.4. Proof of REMARK 0.2. According to 0.2 , only the statement (ii) needs a proof.

Suppose (ii) is false. Then $\gamma([0, \infty)) \subset C_{t}(p)$ and (3.3.1) holds for any $s>0$. It follows that

$$
\varliminf_{s \rightarrow \infty} \frac{\Xi(s)}{s} \geq \varliminf_{s \rightarrow \infty}\left(\frac{\epsilon(R)}{2 \sqrt{2}}-\frac{1}{s}\right) \frac{\pi}{2}=\frac{\pi \epsilon(R)}{4 \sqrt{2}}>0
$$

which contradicts our assumption.

3.5. Proof of TheOREM 1.5. It follows from (3.3.1) and (1.5.1) that

$$
\lambda(t) \leq\left(\frac{A \lambda^{\alpha}(t)}{\pi / 2}+1\right) \frac{2 \sqrt{2}}{\epsilon(R(t))} .
$$

Suppose first that $\alpha \neq 0$. Fix some $\eta>0$. When $t$ (and $\lambda(t)$ ) is sufficiently large, then $\eta A \lambda^{\alpha}(t) /(\pi / 2)>1$. Now by (3.5.1)

$$
\lambda(t) \leq(1+\eta) \frac{A \lambda^{\alpha}(t)}{\pi / 2} \cdot \frac{2 \sqrt{2}}{\epsilon(R(t))} .
$$


Solving for $\lambda(t)$ one has

$$
\begin{aligned}
\lambda(t) & \leq\left((1+\eta) \frac{A 4 \sqrt{2}}{\pi \epsilon(R(t))}\right)^{1 /(1-\alpha)} ; \\
\varlimsup_{t \rightarrow \infty} \frac{\lambda(t)}{z(t)} & \leq\left((1+\eta) \frac{A 2 \sqrt{2}}{\pi} \varlimsup_{t \rightarrow \infty} \frac{2}{\epsilon(R(t)) \cdot z^{1-\alpha}(t)}\right)^{1 /(1-\alpha)} .
\end{aligned}
$$

By Remark 1.2 in [4], $\lim _{t \rightarrow \infty} R(t) / t=1 . z \in Z$ implies that $z^{1-\alpha} \in Z$ i.e.

$$
\lim _{t \rightarrow \infty} \frac{z^{1-\alpha}(R(t))}{z^{1-\alpha}(t)}=1
$$

Then

$$
\begin{aligned}
\varlimsup_{t \rightarrow \infty} \frac{2}{\epsilon(R(t)) \cdot z^{1-\alpha}(t)} & =\varlimsup_{t \rightarrow \infty} \frac{2}{\epsilon(R(t)) \cdot z^{1-\alpha}(R(t))} \cdot \frac{z^{1-\alpha}(R(t))}{z^{1-\alpha}(t)} \\
& =\varlimsup_{t \rightarrow \infty} \frac{2}{\epsilon(R(t)) \cdot z^{1-\alpha}(R(t))}=\Phi_{2}\left(z^{1-\alpha}\right) .
\end{aligned}
$$

(The condition $t \rightarrow \infty$ can be replaced with $R(t) \rightarrow \infty$ since $R(t)$ is a continuous and nondecreasing function going to $\infty$ as $t \rightarrow \infty$.)

Substituting (3.5.3) in (3.5.2) and passing to the limit as $\eta \rightarrow 0$ one obtains (1.5.2) for the case $\alpha \in(0,1)$.

For the case $\alpha=0$, one can obtain (1.5.2) by dividing (3.5.1) by $z(t)$ and calculating the upper limit as above.

Let $p^{*} \in M, \rho\left(p, p^{*}\right)=\Delta>0$ and $t>0$. By 3.1, $B_{t-\Delta}(p) \subset B_{t}\left(p^{*}\right) \subset$ $B_{t+\Delta}(p)$. Therefore

$$
\lambda(t-\Delta) \leq \lambda^{*}(t) \leq \lambda(t+\Delta) .
$$

(We use the notation of 3.1.) Since $z \in Z$, we have

$$
\varlimsup_{t \rightarrow \infty} \frac{\lambda(t \pm \Delta)}{z(t)}=\varlimsup_{t \rightarrow \infty} \frac{\lambda(t \pm \Delta)}{z(t \pm \Delta)} \cdot \lim _{t \rightarrow \infty} \frac{z(t \pm \Delta)}{z(t)}=\varlimsup_{t \rightarrow \infty} \frac{\lambda(t)}{z(t)} .
$$

Dividing (3.5.4) by $z(t)$, passing to the upper limits and applying (3.5.5) one obtains

$$
\varlimsup_{t \rightarrow \infty} \frac{\lambda(t)}{z(t)}=\varlimsup_{t \rightarrow \infty} \frac{\lambda^{*}(t)}{z(t)}
$$

i.e. the upper limit in (1.5.2) does not depend on $p \in N$.

3.6. PROOF OF THEOREM 1.4. By Theorem 2.2, $s(t)$ is not larger than the diameter of $M_{0}$. This and $[4,(1.4)]$ imply that

$$
s(t) \leq \frac{2}{\sqrt{k(R(t))}} \tan ^{-1} \frac{\sqrt{k(R(t))}}{\epsilon(R(t))} .
$$

Just as in 3.5 , we have

$$
\varlimsup_{t \rightarrow \infty} \frac{s(t)}{z(t)}=\varlimsup_{t \rightarrow \infty} \frac{2}{z(R(t)) \cdot \sqrt{k(R(t))}} \tan ^{-1} \frac{\sqrt{k(R(t))}}{\epsilon(R(t))} \cdot \frac{z(R(t))}{z(t)}=\Phi_{1}(z) .
$$

Since $\Phi_{1} \leq \Phi_{2}$ and $\Phi_{1} \leq \Phi_{3}$ (see 1.3), this implies (1.4.1). 
The independence of the upper limit in (1.4.1) from the point $p \in N$ is proved exactly as in 3.5 .

3.7. Proof of Corollary 0.4. Since $t^{\beta} \in Z$ and by Lemma $1.2, h_{\beta}=$ $\Phi_{3}\left(t^{\beta}\right)$ does not depend on $p$. Inequalities (0.4.1) and $\beta \geq 1$ follow from 1.4.

For any $\delta>0, \pi /\left(t^{\beta} \sqrt{k(t)}\right)<h_{\beta}+\delta$ if $t$ is sufficiently large. Hence $k(t)>$ $\pi^{2} /\left(t^{2 \beta}\left(h_{\beta}+\delta\right)^{2}\right)$. By $[4,(1.13 .2)]$,

$$
\epsilon(t)>K(t)=\int_{t}^{\infty} k(x) d x>\pi^{2} / t^{2 \beta-1}\left(h_{\beta}+\delta\right)^{2}(2 \beta-1) .
$$

Therefore $2 /\left(t^{2 \beta-1} \epsilon(t)\right)<2\left(h_{\beta}+\delta\right)^{2}(2 \beta-1) / \pi^{2}$. Passage to the upper limit yields $\Phi_{2}\left(t^{2 \beta-1}\right)<2\left(h_{\beta}+\delta\right)^{2}(2 \beta-1) / \pi^{2}$ and, since $\delta$ is arbitrary, $\Phi_{2}\left(t^{2 \beta-1}\right) \leq$ $2 h_{\beta}^{2}(2 \beta-1) / \pi^{2}$. Obviously, $z=t^{(2 \beta-1) /(1-\alpha)} \in Z$ because $\beta \geq 1$ and $\alpha \in(0,1)$. Hence $\Phi_{2}\left(z^{1-\alpha}\right) \leq 2 h_{\beta}^{2}(2 \beta-1) / \pi^{2}$. Now (0.4.2) follows from (1.5.2).

The upper limits in (0.4.1) and (0.4.2) do not depend on $p$ by Theorems 1.4 and 1.5.

\section{BIBLIOGRAPHY}

1. J. Cheeger and D. Gromoll, On the structure of complete manaifolds of non-negative curvature, Ann. of Math. (2) 96 (1972), 413-443.

2. B. V. Dekster, Estimates of the length of a curve, J. Differential Geom. 12 (1977), 101-117.

3. - Upper estimates of length of a curve in a Riemannian manifold with boundary, J. Differential Geom. 14 (1979), 149-166.

4. B. V. Dekster and I. Kupka, Asymptotics of curvatures in a space of positive curvature, J. Differential Geom. 15 (1981), 553-568.

5. D. Gromoll and W. Meyer, On complete open manifolds of positive curvature, Ann. of Math. (2) 90 (1969), 75-90.

6. Ju. G. Resetnjak, An estimate of the length of a rectifiable curve in an n-dimensional space, Sibirsk. Mat. Ž. 2 (1961), 261-265. (Russian)

Department of Mathematics, University of Notre Dame, Notre Dame, INDIANA 46556

Current address: Department of Mathematics, Erindale College of University of Toronto, Mississauga, Ontario, Canada L5L 1 C6 